\title{
NEUMONÍA NOSOCOMIAL: DEFINICIÓN, DIAGNÓSTICO Y TRATAMIENTO
}

\author{
Salas Segura, Donato \\ Especialista en Medicina Crítica y Cuidado Intensivo del Hospital Rafael Ángel Calderón Guardia. San José, \\ Costa Rica. Profesor de la Escuela de Tecnologías en Salud y del Departamento de Farmacología y Toxicología \\ de la Escuela de Medicina de la Universidad de Costa Rica. San Pedro Costa Rica.
}

\begin{abstract}
Resumen: La neumonía nosocomial es una causa importante de morbi-mortalidad hospitalaria cuyo tratamiento oportuno es fundamental. El diagnóstico de esta entidad puede no ser fácil y se asienta, en buena parte, en un alto índice de sospecha pese a lo cual el tratamiento antibiótico debe ser instaurado rápida y acertadamente con el fin de disminuir la mortalidad.

La escogencia del tratamiento debe hacerse con base en los antecedentes del paciente, a la flora local de cada hospital, a las características farmacológicas de los antibióticos y a los resultados de los cultivos. La adecuada selección de los antibióticos también incluye el correcto y rápido descalonamiento de los mismos.
\end{abstract}

Palabras clave: Neumonía nosocomial, antibióticos

Abstract: Nosocomial pneumonia is a major cause of morbidity and hospital mortality which early treatment is essential. The diagnosis of this entity may not be easy and is based largely on a high index of suspicion and antibiotic treatment should be initiated quickly and accurately in order to decrease mortality. The choice of treatment is made on basis of patient history, local flora of each hospital, the pharmacological characteristics of antibiotics and culture results. Appropriate antibiotic selection also includes adequate and rapid descaled of them.

Key words: Nosocomial pneumonia, antibiotics

Recibido: 20 Septiembre 2011. Aceptado: 16 Octubre 2011. Publicado: 3 Noviembre 2011. 


\section{INTRODUCCIÓN}

La neumonía es la segunda infección nosocomial más frecuente en un hospital general y la infección más frecuente en las unidades de cuidado intensivo [1]. Por definición, se acepta que aparece después de las primeras 48 horas de internamiento y hasta 7 días después del egreso del paciente. Existe también el concepto de neumonía asociada a centros o servicios de salud ("health care-associated pneumonia"), el cual se refiere a la casos de neumonía que aparece en pacientes de riesgo especial como lo son los pacientes que asisten a servicios de hemodiálisis, viven en hogares de ancianos, han estado hospitalizados en los últimos 90 días, recibieron antibióticos endovenosos recientemente o quimioterapia [2,3]. En ambos grupos, los tipos de gérmenes causantes de la infección son, usualmente, virulentos, agresivos y con patrones de resistencia a los antibióticos, lo que les da un factor común. asocia a una estancia del doble de lo normal (14 días versus 7 días) y a una tasa de mortalidad de hasta el $24 \%$.

\section{DIAGNÓSTICO}

Los criterios clínicos para el diagnóstico de neumonía nosocomial (NN) son tan inespecíficos como lo pueden ser la fiebre, la leucocitosis y el aumento de secreciones, pues estas manifestaciones se pueden presentar en varias condiciones pulmonares no necesariamente infecciosas como lo son la atelectasia, la lesión pulmonar aguda o el tromboembolismo pulmonar.

De la misma manera los hallazgos clínicos son sensibles, pero poco específicos, la radiografía de tórax con frecuencia muestra hallazgos que no permiten un diagnóstico definitivo de neumonía [3,4].

Tabla No. 1. Criterios clínicos que permiten la sospecha de neumonía.

A pesar de ser poco específicos, la presencia concomitante de 2 de los siguientes criterios permite establecer una sospecha válida de neumonía nosocomial.

1. Fiebre $>$ de $38^{\circ} \mathrm{C}$, sin otra causa reconocida.

2. Aumento de los requerimientos de oxígeno.

3. Desarrollo de nuevos infiltrados pulmonares o aumento de los previos en la radiografía de tórax.

4. Aumento en el volumen de las secreciones o cambio en sus características (purulento).

La neumonía asociada al ventilador (NAV), es un grupo dentro del espectro de la neumonía nosocomial en el cual el principal de factor de riesgo es la intubación endotraqueal y la pérdida de los mecanismos de defensa de la vía aérea.

La neumonía nosocomial tiene un importante impacto en la morbimortalidad de los pacientes, y en la duración y costos del internamiento. En la Unidad de Cuidados Intensivos del Hospital Calderón Guardia, por ejemplo, el desarrollo de neumonía nosocomial se
Se han desarrollado modelos de predicción basados en diferentes sistemas de puntaje que tampoco han logrado alcanzar valores de especificidad $o$ sensibilidad que los vuelvan una herramienta definitiva en el diagnóstico [5,6].

Por lo tanto no existe un patrón de oro (gold standard) en el diagnóstico de la NN, lo que sin embargo no es óbice para tratar de iniciar el tratamiento antibiótico con la mayor prontitud posible ante la sospecha bien fundada de infección. 
La sospecha clínica de NN obliga a la toma de cultivos de la vía aérea. Un procedimiento ideal, pero escasamente utilizado es el lavado broncoalveolar (LBA), más común es el aspirado traqueal, si el paciente se encuentra intubado. La presencia de $10^{4}$ unidades formadoras de colonias (UFC) o más, obtenidas por LBA o de $10^{5}$ UFC en el aspirado bronquial se considera positivo por infección [7]. Concomitantemente con la toma de los cultivos de la vía aérea se deben tomar 2 hemocultivos de sitios diferentes.

El uso de biomarcadores, especialmente de procalcitonina, es útil para diferenciar infecciones bacterianas de otras condiciones inflamatorias del parénquima pulmonar [8,9], aunque falla cuando se utiliza como un valor diagnóstico aislado para NN [9] y por lo tanto no debe ser utilizado para este propósito. La procalcitonina es útil para el seguimiento de la respuesta al tratamiento antibiótico, se ha demostrado elevada mortalidad que puede comportar la neumonía, especialmente si la antibioterapia es inadecuada [11]. La elección del tratamiento empírico se efectuará teniendo en cuenta diferentes factores, como la flora bacteriana propia del hospital, con sus particulares resistencias antibióticas; antibioterapia previa y factores inherentes del huésped predisponentes a uno $\mathrm{u}$ otro tipo de microorganismo, entre otros. Además, si la infección se ha adquirido en una unidad de cuidados intensivos, hay dos elementos que dificultan la elección del antibiótico para tratar a estos pacientes: por un lado, el posible alto grado de resistencia de los gérmenes, especialmente en pacientes que han recibido antibióticos y, por otro, el que en muchos casos la neumonía es polimicrobiana $[12,14]$. En cualquier caso, el tratamiento empírico deberá ajustarse a los resultados microbiológicos en cuanto se disponga de estos.

Tabla No.2 ¿Qué hallazgos radiológicos permiten establecer un diagnóstico de probabilidad de neumonía nosocomial?

Aunque con frecuencia los hallazgos radiológicos de la placa de tórax son inespecíficos, hay algunos de ellos que son de gran utilidad siempre y cuando se presenten en dos o más radiografías:

1. Existencia de un infiltrado nuevo o progresivo ó bien persistente.

2. Consolidaciones.

3. Cavitaciones.

En ausencia de una enfermedad pulmonar o cardiaca de fondo (p.ej. enfermedad pulmonar obstructiva crónica, edema pulmonar, o lesión pulmonar aguda) una sola radiografía con los cambios descritos es una opción aceptable.

que si los niveles de procalcitonina se mantienen altos luego de 3 días de terapia antibiótica esto constituye un marcador de falla terapéutica y del mismo modo, la disminución de los niveles de procalcitonina, junto con la mejoría clínica, permiten abreviar la duración del tratamiento antibiótico [10].

\section{TRATAMIENTO}

El tratamiento antibiótico deberá administrarse inicialmente de forma empírica, lo que debe efectuarse además de la forma más precoz posible, dada la
A manera de ejemplo de las peculiares variedades de la flora local, en la tabla No.3 se muestran los diez gérmenes más frecuentemente aislados en NN en la Unidad de Cuidados Intensivos del Hospital Calderón Guardia durante un periodo de 5 años. Ante este patrón de distribución bacteriano, es válido iniciar tratamientos empíricos dirigidos fundamentalmente contra bacterias gram negativas (BGN) entéricos (Enterobacter, E. Coli, Klebsiella) y estafilococos sin iniciar cobertura anti-pseudomónica. 
Tabla No. 3. Gérmenes más frecuentemente aislados en neumonía nosocomial en la Unidad de Cuidados Intensivos del Hospital Calderón Guardia entre octubre de 2006 a septiembre de 2011.

\begin{tabular}{cc} 
Germen & $\begin{array}{c}\text { No. de } \\
\text { aislamientos }\end{array}$ \\
\hline Enterobacter cloacae & 15 \\
Staphylococcus aureus & 14 \\
Klebsiella pneumonie & 14 \\
Pseudomonas aeruginosa & 9 \\
Escherichia coli & 7 \\
Staphylococcus epidermidis & 6 \\
Serratia marcescens & 4 \\
Enterobacter aerogenes & 2 \\
Acinetobacter spp & 2 \\
Citrobacter freundii & 2 \\
\hline
\end{tabular}

Fuente: Bases de datos UCI-HCG

Es necesario recordar que para iniciar el tratamiento empírico la duración de la hospitalización o período de tiempo transcurrido desde el ingreso hasta el inicio de la neumonía es importante, porque desde este punto de vista existen dos categorías que permiten clasificar la neumonía nosocomial, una en precoz, cuando se presenta antes de los primeros 5 días de hospitalización, y en neumonía nosocomial tardía, si se presenta después del quinto día del ingreso hospitalario. En el segundo caso, la presencia de Pseudomonas aeruginosa, especies de Acinetobacter y de Staphylococcus aureus meticilino resistente (MRSA,

Tabla No.4 ¿Cuáles son los factores más importantes que deben ser tomados en cuenta para hacer la selección óptima inicial del tratamiento antibiótico?

Son 4 factores [13]:

1. La sospecha sobre cuál es el germen casual y sus posibles patrones de sensibilidad basado en el cuadro clínico y en estudios de la flora bacteriana local

2. Los datos obtenidos en cultivos de vigilancia en el mismo paciente.

3. Los hallazgos en el frotis del material del aspirado bronquial.

4. La actividad antibacteriana intrínseca de los agentes antimicrobianos y sus características farmacocinéticas. 
por sus siglas en inglés) es mucho más frecuente que en el grupo temprano en el que predominan BGN entéricas y estafilococos meticilino sensibles.

\section{¿Cuál debe ser la terapia antibiótica empírica inicial?}

La terapia antibiótica inicial debe tomar en cuanta dos factores, el primero es el momento de aparición de la neumonía, esto es, si es temprana o tardía, y el segundo, es si el paciente se encontraba recibiendo antibióticos previamente (por cualquier causa) o no. vancomicina (como estafilococcida).

\section{Duración de la terapia antibiótica}

La duración de la terapia antibiótica deberá individualizarse en cada caso, dependiendo de la gravedad de la enfermedad, la rapidez de la respuesta clínica y del microorganismo causal. La mayoría de las neumonías producidas por BGN entéricas pueden tratarse en forma segura con solamente 7 días. Si el patógeno causal es $S$. aureus meticilino sensible, una duración de 7 a10 días puede ser suficiente. En los casos en que la neumonía sea multilobar, existan malnutrición, mal estado general o neumonía

\section{¿Qué significa el término "descalonar" ?}

La palabra descalonar es un anglicismo que se ha adaptado al lenguaje médico en castellano como sinónimo de ajuste en la terapia antibiótica. El concepto básico radica en el hecho de que, una vez identificado el germen causante de una infección (urinaria, respiratoria, etc.) es posible estrechar el tratamiento antibiótico a la menor cantidad de drogas sin que esto resulte perjudicial para el paciente y por el contrario, se disminuyan el número de reacciones adversas a antibióticos, el surgimiento de cepas bacterianas resistentes, y los costos de internamiento.

Si la neumonía es temprana y el paciente no ha recibido antibióticos entonces se debe iniciar con una cefalosporina de tercera generación sin actividad antipseudomónica (cefotaxima por ejemplo y costumbre) o un betalactámico asociado a un inhibidor de betalactamasa (p.e amplicilina con sulbactam).

Por otra parte, si la neumonía es temprana y el paciente ha recibido antibióticos o si es tardía pero el paciente no ha recibido antibióticos se debe iniciar con un aminoglicósido o una quinolona asociados con ceftazidima (una cefalosporina de tercera generación con actividad anti-pseudomónica) o cefepima (una cefalosporina de cuarta generación) o piperacilinatazobactam o ticarcilina-clavulonato.

Si la neumonía es tardía y el paciente ha recibido antibióticos, el tratamiento deberá incluir un aminoglicósido (una quinolona es una opción válida), un carbapenémico (meropenem o imipenem) y necrosante por BGN o cavitación radiológica y también

en aquellos casos en que el germen causal sea $P$. aeruginosa o Acinetobacter spp., se aconseja una duración de 14 a 21 días de tratamiento $[13,14,15]$.

El cambiar de vía intravenosa a la vía oral luego de unos días para completar el tratamiento es apropiado y válido en aquellos casos en que el organismo infectante sea susceptible in vitro al antibiótico que quiera administrarse por vía oral, la mejoría clínica sea evidente, y exista una absorción oral adecuada [16].

\section{Terapia de descalonamiento}

Una vez que se dispone de los resultados de los cultivos es necesario descalonar la terapia antibiótica para evitar el uso prolongado de un tratamiento 
antibiótico de amplio espectro, que además de caro, es capaz de producir el surgimiento de cepas resistentes [17]. Aun en pacientes con estancias hospitalarias prolongadas, es relativamente frecuente el asilamiento de cepas bacterianas que no son altamente resistentes por lo que es posible descalonar la terapia a un solo antibiótico $[18,19,20]$.

\section{REFERENCES}

1. Chastre J, Fagon JY. Ventilator-associated pneumonia. Am J Respir Crit Care Med 2002;165:867-903.

2. Morrow LE, Kollef MH. Recognition and prevention of nosocomial pneumonia in the intensive care unit and infection control in mechanical ventilation. Crit Care Med 2010 Vol. 38, No. 8 (Suppl.): S352S362.

3. American Thoracic society, Infectious Diseases Society of America: Guidelines for the management of adults with hospital acquired, ventilator-associated, and healthcareassociated pneumonia. Am J Respir Crit Care Med 2005; 71:388-416.

4. Mandell LA, Wunderink RG, Anzueto A, et al: Infectious Diseases Society of America/ American Thoracic Society consensus guidelines on the management of communityacquired pneumonia in adults. Clin Infect Dis 2007; 44:S27S72.

5. Luyt CE, Chastre J, Fagon JY: Value of the clinical pulmonary infection score for the identification and management of ventilator- associated pneumonia. Intensive Care Med 2004; 30:844-852 22.

6. Croce MA, Swanson JM, Magnotti LJ, et al. The futility of the clinical pulmonary infection score in trauma patients. $J$ Trauma 2006; 60:523-527.

7. Torres A, Fabregas N, Ewig S, et al: Sampling methods for ventilator-associated pneumonia: Validation using different histologic and microbiological references. Crit Care Med 2000; 28:2799-804.

8. Christ-Crain M, Muller B: Biomarkers in respiratory tract infections: diagnostic guides to antibiotic prescription, prognostic markers and mediators. Eur Respir J 2007; 30:556-573.

9. Ip M, Rainer TH, Lee N, et al: Value of serum procalcitonin, neopterin, and C-reactive protein in differentiating bacterial from viral etiologies in patients presenting with lower respiratory tract infections. Diagn Microbiol Infect Dis 2007; 59:131-136.

10. Christ-Crain M, Stolz D, Bingisser R, et al: Procalcitonin guidance of antibiotic therapy in community-acquired pneumonia: A randomized trial. Am J Respir Crit Care Med 2006; 174:84-93.

11. Alvarez-Lerma F. Modification of empiric antibiotic treatment in patients with pneumonia acquired in the intensive care unit. ICU-Acquired Pneumonia Study Group. Intensive Care Med 1996; 22:387-94.

12.Trouillet JL, Chastre J, Vuagnat A, et al. Ventilatorassociated pneumonia caused by potentially drug-resistant bacteria. Am J Respir Crit Care Med 1998; 157:531-539.

13. Chastre J. Antimicrobial treatment of hospital-acquired pneumonia. Infect Dis Clin N Am 2003; 17: 727-737.

14. Ibrahim EH, Ward S, Sherman G, et al: Experience with a clinical guideline for the treatment of ventilator-associated pneumonia. Crit Care Med 2001; 29:1109-1115

15. Hanberger H, Garcia-Rodriguez JA, Gobernado M, et al. Antibiotic susceptibility among aerobic gram-negative bacilli in intensive care units in 5 European countries. French and Portuguese ICU Study Groups. JAMA 1999; 281:67-71.

16. Dennesen PJ, van der Ven AJ, Kessels AG, et al: Resolution of infectious parameters after antimicrobial therapy in patients with ventilator- associated pneumonia. Am J Respir Crit Care Med 2001; 163:1371-1375.

17. Hoffken G, Niederman MS. Nosocomial pneumonia: the importance of a de-escalating strategy for antibiotic treatment of pneumonia in the ICU. Chest 2002; 122: 21832196.

18. Chastre J, Wolff M, Fagon JY, et al. Comparison of 8 vs 15 days of antibiotic therapy for ventilator-associated neumonia in adults. A randomized trial. JAMA 2003; 290:2588-2598. 
19. Fink MP, Snydman DR, Niederman MS, et al. Treatment of severe pneumonia in hospitalized patients: results of a multicenter, randomized, double-blind trial comparing intravenous ciprofloxacin with imipenem-cilastatin. The Severe Pneumonia Study Group. Antimicrob Agents Chemother 1994; 38:547-57.

20. Micek ST, Ward S, Fraser VJ, et al: A randomized controlled trial of an antibiotic discontinuation policy for clinically suspected ventilator-associated pneumonia. Chest 2004; 125:1791-1799.

\section{INFORMACION DE AUTOR:}

Donato A. Salas-Segura.

Apdo. Postal 10706-1000 SJ, Costa Rica

e-mail: dasscom@racsa.co.cr 